\title{
Nanostructural and electrical properties of functionally terminated self-assembled monolayers on silicon surfaces
}

\author{
M. Rittner \\ 4. Physikalisches Institut, Universitaet Stuttgart, Pfaffenwaldring 57, 70550 Stuttgart, Germany
}

\section{S. Martin-Gonzalez}

Consejo Superior de Investigaciones Cientificas, Centro Nacional de Microelectronica, C/Isaac Newton 8, 28760 Tres Cantos, Madrid, Spain

\author{
A. Flores \\ Consejo Superior de Investigaciones Cientificas, Instituto de Estructura de la Materia, C/Serrano 121, \\ 28006 Madrid, Spain \\ H. Schweizer \\ 4. Physikalisches Institut, Universitaet Stuttgart, Pfaffenwaldring 57, 70550 Stuttgart, Germany \\ F. Effenberger \\ Institut fuer Organische Chemie und Isotopenforschung, Universitaet Stuttgart, Pfaffenwaldring 55, \\ 70550 Stuttgart, Germany \\ M. H. Pilkuhna) \\ 4. Physikalisches Institut, Universitaet Stuttgart, Pfaffenwaldring 57, 70550 Stuttgart, Germany and \\ Consejo Superior de Investigaciones Cientificas, Instituto de Estructura de la Materia, C/Serrano 121, \\ 28006 Madrid, Spain
}

(Received 28 July 2004; accepted 25 July 2005; published online 12 September 2005)

Self-assembled monolayers (SAMs) having alkyl chains with typically $18 \mathrm{CH}_{2}$ units and with different functional end groups (methyl, thiol, thiophene, phenoxy, and biphenyl) have been attached to hydroxylated (100) silicon surfaces. Their layer structure has been studied using grazing incidence $\mathrm{x}$-ray reflectometry. An excellent data analysis is possible on the basis of a two layer model. One layer with constant thickness (about $10.8 \AA$ A) for all the SAMs investigated is associated with the alkyl chain/silicon interface, whereas the second layer is associated with the functional end group. Its dimension changes with the size and nature of the end group. The layer dimension increases from about $22 \AA$ for the smallest end group (methyl) to about $32 \AA$ for the largest one (biphenyl). The experimental layer thickness values are in good agreement with those expected from molecular modeling. The electrical properties of the SAM layers have been studied using Au/Al contacts deposited on the functional end groups. Of particular interest are the insulating properties of the alkyl chain and the breakdown voltages which exhibit very high values of typically $16 \mathrm{MV} / \mathrm{cm}$. A lateral in-plane conductance along the end groups has been measured in the case of an $\mathrm{I}_{2}$-doped biphenyl end group. Iodine doping can increase the conductivity by a factor of 12-14. This suggests the possibility of a nanomolecular transistor with the functional end group as an active layer without any additional deposition of an organic conducting layer on the SAM dielectric layer. (c) 2005 American Institute of Physics. [DOI: 10.1063/1.2035309]

\section{INTRODUCTION}

Ultrathin organic self-assembling monolayers (SAMs) on semiconductor or metal surfaces have received considerable attention as nanostructures in recent years. ${ }^{1,2}$ In the case of metal substrates, thiols on Au have become a representative key system ${ }^{2}$ with the occurrence of long-range order, for instance, in organic octanethiol molecules on $\mathrm{Au}$ (111) surfaces. ${ }^{3}$

In the case of semiconductor substrates, silicon surfaces with their potential for combining semiconductor nanoelectronics with organic molecular electronics are of particular interest. Frequently studied systems are OTS (octadecyltrichlorosilane: $\left.\mathrm{CH}_{3}-\left(\mathrm{CH}_{2}\right)_{18}-\mathrm{SiCl}_{3}\right)$ reacting with hydroxy-

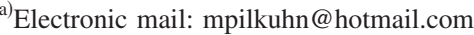

lated Si surfaces. ${ }^{4-8}$ The reaction of the $\mathrm{SiCl}_{3}$ "anchor group" leads to a chemical bond between the organic molecules and the substrate. The molecules are also bound to each other according to the Si-O-Si network of the substrate ${ }^{9}$ by van der Waals forces. In general, the entropy loss during ordering should be compensated by an enthalpy gain. This enthalpy gain is given by the reaction of the anchor group with the substrate and the van der Waals interaction between neighboring alkyl chains which leads to typical $\left(\mathrm{CH}_{2}\right)_{n}$ chain lengths with $12<n<18$. $^{10}$

Whether a complete full large area monolayer coverage with ordered molecules can be reached depends on experimental conditions. ${ }^{11,27}$ Because of a possible steric hindrance, ${ }^{12,13}$ long-range ordering of molecules may be limited to domainlike smaller areas. But there are examples, for instance, that phenoxy terminated monolayers (PhO-OTS 


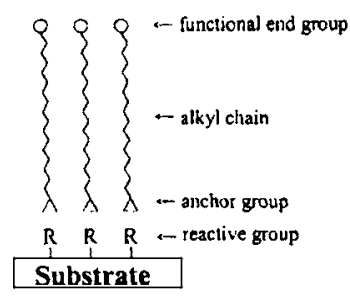

Si (100) substrate

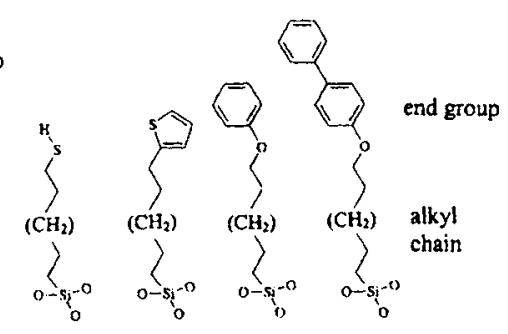

(a)

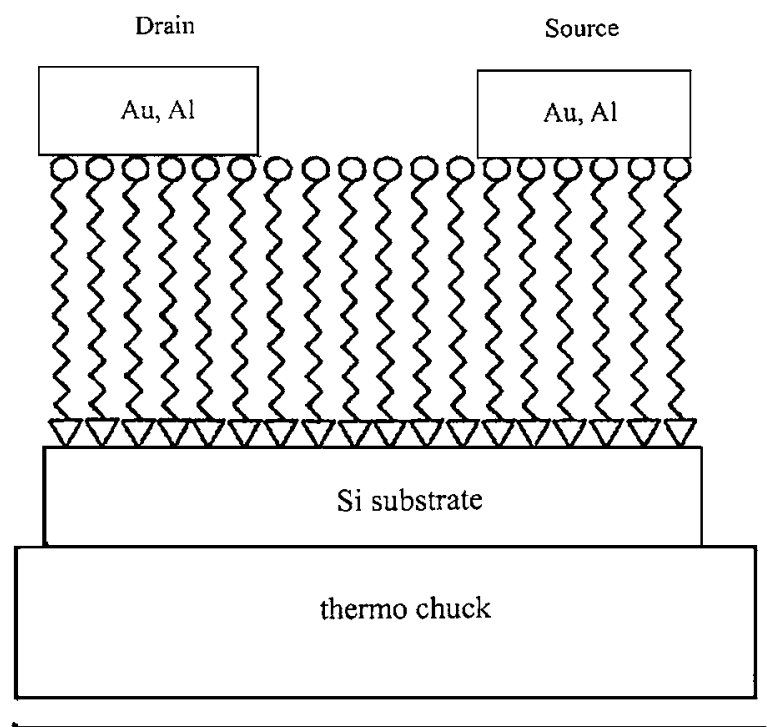

(b)

FIG. 1. (a) Attachment of SAM layers through a $\mathrm{CH}_{3}$ anchor group to a hydroxylated silicon (100) surface. The SAM molecules contain an alkyl chain with constant length $\left(\mathrm{CH}_{2}\right)_{18}$ and different functional end groups which are (from left to right) thiol, thiophene, phenoxy, and biphenyl. (b) Transistorlike structure for electrical characterization.

SAMs) are continuous and defect (pin-hole)-free over large areas without exhibiting domain boundaries. ${ }^{27}$ In general, tilt angles between the substrate and the molecules have to be assumed. ${ }^{6,14}$ In the case of OTS on hydroxylated $\mathrm{Si}(100)$ surfaces, they seem to be small, i.e., between $10^{\circ}$ and $20^{\circ}{ }^{2,6}$ SAM layers can be also obtained on oxide-free $\mathrm{H}$ terminated $\mathrm{Si}(111)$ surfaces. ${ }^{14,15}$ In this case, the monolayers form a $\mathrm{C}-\mathrm{Si}$ bond at the surface.

In view of possible applications, OTS-type SAMs have excellent insulating properties, which makes them attractive for nanoelectronic field-effect devices as gate dielectrics. ${ }^{16-18,27}$

In this paper, SAM layers on hydroxylated Si surfaces with molecules having functional aromatic end groups at the end of the alkyl chain are studied. The reaction of a $\mathrm{SiCl}_{3}$ anchor group connects the alkyl chain, which is typically $\left(\mathrm{CH}_{2}\right)_{18}$, with the silicon surface.

The end groups could be varied, and they were thiol, thiophene, phenoxy, and biphenyl [Fig. 1(a)]. The methyl terminated $\left(\mathrm{CH}_{2}\right)_{18}$ system, for simplicity also called OTS, served as a reference.

These systems allowed the evaporation of $\mathrm{Au}$ and $\mathrm{Al}$ contacts on the functional end groups which, like in the case of the biphenyl end groups, tightly closed off the ordered alkyl chains. Migration of metal atoms between the chains was not noticed, and in this way the insulating property of the chains can be maintained. With a lateral in-plane conductivity along the biphenyl functional end group, a new type of nanotransistor without additional conducting layers such as pentacene as an active layer ${ }^{27}$ is possible. This is schematically shown in Fig. 1(b), where the functional end group serves as an active layer, the two gold contacts as source and drain, and the silicon wafer as a gate electrode.

\section{EXPERIMENT}

\section{A. SAM preparation on hydroxylated silicon surfaces}

Clean silicon surfaces with low surface roughness were essential for the SAM preparation.

The following two step wet etching procedure on polished (100) oriented $\mathrm{Si}$ wafers was used: $\mathrm{H}_{2} \mathrm{SO}_{4}(96 \%)$ : $\mathrm{H}_{2} \mathrm{O}_{2}(31 \%)$ with ratio 4:1 (Piranha acid) for 30-60 min at $90{ }^{\circ} \mathrm{C}$ followed by $\mathrm{HF}(0.5 \%)$ and isopropyl alcohol $(0.1 \%) .{ }^{19}$ This resulted in hydroxylated surfaces. Alternatively, $\mathrm{OH}$ groups on the silicon surface were obtained by exposing the surface to an oxygen plasma. A surface roughness of $2-4 \AA$ as determined by grazing incidence $\mathrm{x}$-ray reflectometry.

Molecular ordering of the alkyltrichlorosilane molecules (with appropriate aromatic end groups) was achieved either from solution or from the vapor phase. ${ }^{14}$

After chemisorption, the $\mathrm{SiCl}_{3}$ anchor groups reacted with the $\mathrm{OH}$ groups of the hydroxylated surfaces under development of $\mathrm{HCl}$ leading to the configuration shown in Fig. 1(a). In the case of the solution process, an anhydrous toluene solution was used, and molecular self-assembly occurred within a short time at room temperature. A higher temperature was used for the vapor phase process. In that case, the substrate was kept with the organic molecules in a lowpressure inert gas. ${ }^{14,27}$ The resulting SAM layer had a thickness of approximately $2.5 \mathrm{~nm}$.

For lateral in-plane conductivity investigation, SAMs with biphenyl endgroups were chosen because the $\pi$ - $\pi$ interaction of neighboring molecules leads to close packaging at the surface. Both iodine and $\mathrm{FeCl}_{3}$ doping experiments were made. In the case of iodine doping, $\mathrm{I}_{2}$ was placed together with the sample into a closed quartz tube. The tube was heated to $70{ }^{\circ} \mathrm{C}$ for $1 \mathrm{~h}$ allowing the $I_{2}$ to evaporate to a reactive atmosphere, and to react with the surface layer. Although the exact iodine concentration in the biphenyl top layer is not known, this doping procedure qualitatively leads to reproducible conductivity (cf. Fig. 5).

The $\mathrm{FeCl}_{3}$ experiments, where an evaporated layer was deposited on the sample surface, were not so successful.

\section{B. Structural investigation}

The structural characterization of the various SAM layers in the direction normal to the surface layer was carried out by means of grazing incidence $\mathrm{x}$-ray diffraction, using a Philips X-PERT four cycle diffractometer. The $2 \theta / \omega$ scans were performed in the range $2 \theta=-0.4^{\circ}-10^{\circ}$. The reflectance 
spectra obtained were fitted with a simulation software which includes a fit optimization algorithm to determine the layer thickness and the surface roughness.

\section{Electrical characterization and contacts}

Great care was taken in producing suitable microstructured contacts by lithography or shadow mask deposition. Au contact layers were deposited by $e$-beam evaporation at background pressures of $<10^{-7}$ mbar. Al contacts were made by thermal evaporation at background pressures of $<2$ $\times 10^{-6} \mathrm{mbar}$ at an evaporation rate of about $8 \AA / \mathrm{s}$. For improvement of the gold contact adherence, a 5-nm-thick $\mathrm{Cr}$ buffer layer was introduced which was promising in the case of the biphenyl terminated SAM. The distance between Au contacts could be reduced to about $5 \mu \mathrm{m}$. For the electrical measurements it was essential to avoid contact damage by a contact needle. Good results were obtained with a "soft" tungsten contact needle with a spherical tip of typically $20-\mu \mathrm{m}$ diameter.

An identical reference metal contact was always made on the uncovered hydroxylated silicon surface, and its current-voltage characteristic was compared with that of a contact on the SAM covered surface [compare Fig. 4(a)]. The very much lower current densities for SAM covered surfaces suggest that tunneling is the prevailing mechanism and not "leakage currents" via pin holes or a shunt current path via metal atoms which have migrated between the alkyl chains.

For contacting the back side of the Si wafer, the SAM layer deposited there had to be, first mechanically removed and then, treated with HF.

For dc measurements, the samples were attached to a sample holder with controlled temperature (down to $-60{ }^{\circ} \mathrm{C}$ ) which was at zero ground potential. A screened Kelvin-type setup allowed dark current measurements down to $10 \mathrm{fA}$. Various integration times were used with a HP 4156B system, the longest $(>320 \mathrm{~ms}$ ) for sensitive lateral conductivity measurements.

\section{STRUCTURAL PROPERTIES}

\section{A. Grazing incidence x-ray reflectometry}

$\mathrm{X}$-ray reflectometry based on total reflection characterizes the electron density profile normal to the sample surface. For this reason, this technique was employed to study the structural properties of the SAM layers, with different functional end groups, chemically attached to a hydroxylated $\mathrm{Si}$ (100) surface. A computer data analysis yields data about layer thicknesses, ${ }^{20}$ surface roughness, and real and imaginary parts of the complex refractive index $n$

$$
n=1-\delta-i \beta,
$$

where $\delta$ is related to the electron density, and $\beta$ to the mass absorption coefficient.

For all layers with different end groups, $\delta$ is positive and very small, which implies that the critical angle for total reflection, $\theta_{C}$, is also very small

$$
\theta_{C}=\sqrt{2 \delta}<0.1^{\circ} \text {. }
$$

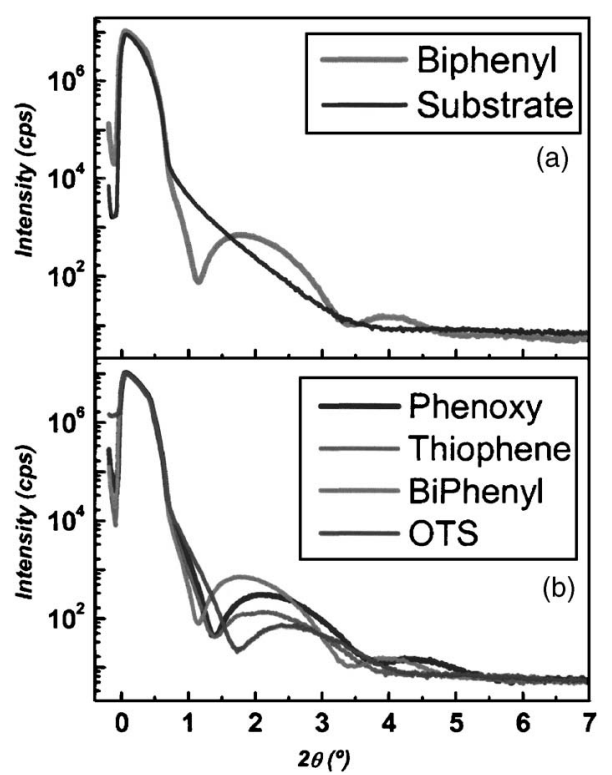

FIG. 2. (a) Grazing incidence x-ray reflectometry: comparison between a silicon substrate with a SAM covered surface having a biphenyl end group. (b) Systematic change of $\mathrm{x}$-ray reflection curves for different SAM covered surfaces: influence of the functional end group.

Figure 2(a) shows a comparison between the grazing incidence x-ray reflection scan of a reference silicon surface with that of a Si surface covered with a SAM layer having a biphenyl end group. The latter exhibits a pronounced interference pattern due to Kiessig interferences of the SAM layer on top of the substrate, while only Fresnel reflectivity is observed for the bare substrate.

Figure 2(b) illustrates the x-ray reflection scans of the different SAM layers with various end groups: biphenyl, phenoxy, thiophene, and OTS (methyl end group). One can see that there is a surprising and very systematic change in the structure of the reflection curves which seems to be related with the size and/or nature of the end group molecule: the larger the end group molecule, the more pronounced the interferences are.

Analysis of the spectra shown in Fig. 2(b) revealed that the data could not be fitted by assuming a one layer model. An excellent fit, however, was obtained with a two layer model, considering that the layers display two different refractive indices $n_{1}$ and $n_{2}$.

Table I collects the thickness values for both layers, layer 1 and layer 2, obtained from the computer analysis. The thinner of the two layers (called layer 1, with $n_{1}$ ) is nearly constant in its dimension, independent of the type of the end group molecule, and its average value is about $10.8 \AA$. This layer is the closest to the substrate.

On the other hand, the thickness of layer 2 (with $n_{2}$ ) varies in the range of $22-32 \AA$ (see Table I). Its dimension depends on the type and size of the end group molecule. Hence, the sum of the two layer thicknesses changes accordingly (see Table I).

The surface roughness obtained from the data analysis in the two layer model varied between 2.9 and $4.5 \AA$ for the thinner layer 1, and between 2.3 and $3.8 \AA$ for the thicker layer 2 . 
TABLE I. Experimental thickness values (layer 1, layer 2, and total thickness) determined from the analysis of the x-ray reflectometry data, together with the simulated SAM thicknesses, denoted as "layer 2 (simulated)," for the various SAMs with different end groups. The corresponding breakdown field values for the case of $\mathrm{Au}$ and $\mathrm{Al}$ contacts are also included.

\begin{tabular}{|c|c|c|c|c|c|c|}
\hline \multirow[b]{3}{*}{ SAM } & \multicolumn{4}{|c|}{ Thickness $(\AA)$} & \multicolumn{2}{|c|}{ Breakdown field $(\mathrm{MV} / \mathrm{cm})$} \\
\hline & Layer 1 & Layer 2 & Total & Layer 2 & Au contact & $\mathrm{Al}$ \\
\hline & \multicolumn{4}{|c|}{ (simulated) } & \multicolumn{2}{|c|}{ contact } \\
\hline OTS & 10.7 & 22.6 & 33.3 & 21.1 & 15.4 & 9.5 \\
\hline Thiophene & 10.9 & 26.5 & 37.4 & 25.1 & $\cdots$ & 9.5 \\
\hline Phenoxy & 10.9 & 26.4 & 37.3 & 26.0 & 13.0 & 6.6 \\
\hline Biphenyl & 10.7 & 32.4 & 43.1 & 29.8 & 11.1 & 20.0 \\
\hline
\end{tabular}

The real part of the refractive index, $\delta$, takes values of $\delta \approx 69 \times 10^{-6}$ for layer 1 , and $\delta \approx 30 \times 10^{-6}$ for layer 2 . Corresponding typical values for the imaginary part, $\beta$, are $\beta$ $\approx 3 \times 10^{-6}$ for layer 1 and $\beta \approx(5-7) \times 10^{-6}$ for layer 2 .

\section{B. Theoretical model and discussion}

Since the x-ray reflection data of the silicon surface do not exhibit Kiessig interferences [compare the two scans included in Fig. 2(a)], the following model is proposed which associates the two layers of the SAM covered surface with the structure of the SAM molecule and an interface:

Layer 1 , with a thickness value of $\approx 11 \AA$ (see Table I) is associated with the interface between the hydroxylated silicon surface and the alkyl chain ("interface layer"). As one would expect, the thickness of this layer does not significantly vary between the different SAMs.

Layer 2, with a thickness value within the range of 22-33 $\AA$ depending on the type of the SAM (see Table I), is associated with the organic monolayer itself, including the functional end group and the alkyl chain or part of it. In this case, the layer thickness increases as the size of the end group increases.

In order to check the validity of this model, we have independently estimated the thickness of each SAM using molecular modeling (MOPAC VI: AM1 package, available through Cerius 2). The simulation of the film thickness is based on the optimization of the geometrical variables of an isolated molecule. As a first approximation, we have assumed that the tilt angle between the molecular axis and the silicon surface is approximately zero. Figure 3 illustrates, as an example, the optimized molecular conformation for the case of the biphenyl end group. The alkyl sequence adopts the all-trans conformation, in agreement with the generally accepted conformation of OTS on oxidized silicon. ${ }^{2}$ Table I collects the molecular length values (measured in the direction of the alkyl sequence chain axis) for each SAM, calculated using molecular modeling.

A good agreement is found between the simulated layer 2 values and the experimental layer 2 thicknesses shown in Table I. Moreover, the delta values determined for layer 2 $\left(\delta \approx 30 \times 10^{-6}\right)$ are substantially different from those obtained for layer $1\left(\delta \approx 69 \times 10^{-6}\right)$. The latter ones are in the range of the $\delta$ values reported for $\mathrm{SiO}_{2}\left(56 \times 10^{-6}\right)$, $\mathrm{Si}(74$

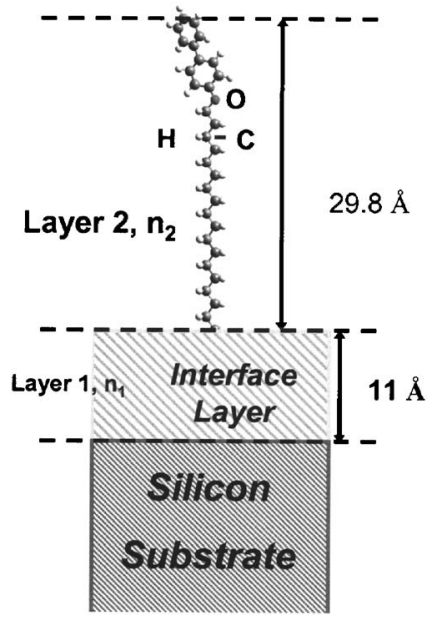

FIG. 3. Theoretical model of two different layers (SAM and interface) for the case of a biphenyl end group.

$\left.\times 10^{-6}\right)$, and C:O:H-based layers $\left(63 \times 10^{-6}\right),{ }^{21}$ in agreement with the concept of an interface layer. Finally, it should be mentioned that preliminary atomic force microscopy (AFM) scratch experiments on the surface of the OTS sample seem to yield a layer thickness value in rough agreement with the experimentally determined layer 2 thickness value reported in Table I. ${ }^{26}$ The above arguments support the concept that layer 2 is associated with the end group terminated alkyl chain (see Fig. 3) describing the molecular structure of the SAM molecule, while layer 1 is related to the interface between the alkyl chain and the silicon surface.

Finally, it should be mentioned that in many cases preliminary AFM images suggest that no long-range order of the SAM molecules occurs, but rather a domainlike coverage. The extension of the domains is $20-50 \mathrm{~nm}$ for OTS and $20-40 \mathrm{~nm}$ for the biphenyl end group. ${ }^{26}$

\section{ELECTRICAL PROPERTIES}

The electrical properties of the SAM layers were investigated with a microstructured contact arrangement, as depicted in Fig. 1(b). For that purpose, Au and $\mathrm{Al}$ contacts were evaporated on the functional end groups in the manner described above. The silicon wafer had an Al contact on the rear side. Both dc and ac measurements were performed.

The main points of interest are (a) the insulating properties of the alkyl chains with the possibility of tunneling or leakage currents and (b) a lateral conductance along the functional end groups.

\section{A. Insulating properties of the alkyl chains with different end groups}

Figure 4(a) shows the current density-voltage characteristics for Au contacts and highly doped $n$-type silicon substrates. Au contacts on hydroxylated silicon surfaces without SAM layers served as a reference. The current densities with SAM coverage are by up to seven orders of magnitude lower compared with the uncovered silicon surface. The lowest 

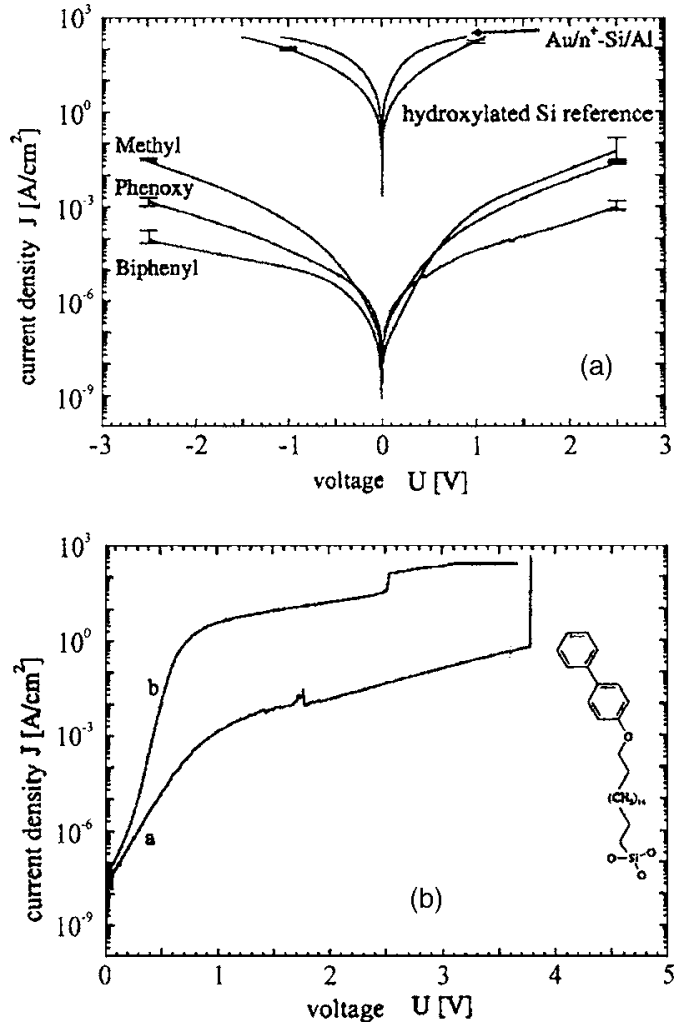

FIG. 4. (a) Vertical current voltage characteristics for silicon covered with SAMs having different end groups. For comparison, the uncovered silicon surface characteristic at much higher current densities is also shown. (b) Current-voltage characteristic with dielectric breakdown for the case of a biphenyl end group (curve a). Measurement after breakdown (curve b).

current density is observed for the case of the biphenyl end group. Al contacts yield somewhat different results, but the observation is qualitatively the same. ${ }^{22,23}$

The mechanism of the extremely low current density through the thin SAM layers is predominantly tunneling, possibly including interface states. The tunneling currents should depend exponentially on the total layer thickness values reported in Table I, according to the WKB approximation. ${ }^{28}$ This is, in fact, observed for phenoxy terminated SAM layers and $\mathrm{Al}$ contacts where alkyl chains with different lengths $(n=6, n=12, n=17)$ were studied. ${ }^{24}$ The influence of the SAM layer thickness can be qualitatively seen clearly in Fig. 4(a): At a fixed voltage, the increase in thickness-by comparing, e.g., the methyl terminated chain with the phenoxy terminated and the biphenyl terminated one-leads to a strong reduction in current density, which is also indicative of tunneling. For a more quantitative modeling the WKB approximation for the current density has been used: ${ }^{28}$

$$
\begin{aligned}
& J=A \exp \left[-(4 \pi / h) d\left(2 m^{*} E_{b}\right)^{1 / 2}\right], \\
& A=\left(2 q^{2} V / h^{2}\right)\left(2 m^{*} E_{F}\right)^{1 / 2} \\
& \left(d=\text { layer thickness, } E_{b}=\right.\text { barrier height, } \\
& V=\text { applied voltage, } E_{F}=\text { Fermi energy } .
\end{aligned}
$$

A comparison for low applied voltages $V$ of the experimentally determined decrease in current density with increasing layer thickness with theory is possible, if the thickness values determined from x-ray reflectometry in Table I are used. As an example, at a fixed voltage of $2,5 \mathrm{~V}$, the ratio of current densities of the methyl terminated chain to that of the biphenyl terminated one is about $2 \times 10^{3}$. The ratio on the basis of the WKB approximation is somewhat larger, namely, about $10^{4}$. Considering the limits of the thickness data, and that there may be differences in the ordering or the interface states, this seems to be a satisfactory agreement.

Of further interest are the breakdown fields which are expected to be large. ${ }^{25,27}$ An example for the case of the biphenyl end group is given in Fig. 4(b), where breakdown occurs at $3.8 \mathrm{~V}$ (curve a). After breakdown, the currentvoltage characteristic has changed to higher current densities (curve b), which means that irreversible changes have occurred during breakdown. A similar behavior was observed for the other SAM layers with different end groups (results not shown here).

Table I shows the breakdown field values, determined using the thickness values (total thickness) obtained from $\mathrm{x}$-ray reflectometry. These fields are, in fact, very large as already reported, ${ }^{27}$ and they are listed in Table I. An average value for the case of Au contacts is about $16 \mathrm{MV} / \mathrm{cm}$. They seem to depend on the type of the end group and the contact metal. An exceptionally large breakdown field of $20 \mathrm{MV} / \mathrm{cm}$ for the biphenyl terminated SAM layer may be somewhat fortuitous. These fields have to be compared with those for $\mathrm{SiO}_{2}$ which are typically $8.5 \mathrm{MV} / \mathrm{cm}^{29}$ This means that the aromatic group terminated SAM layers could serve as excellent insulating layers in silicon nanotechnology as pointed out before. ${ }^{16,18}$

\section{B. Lateral conductance along the functional end groups}

A lateral conduction along the functional end groups is of particular interest for the realization of a molecular transistor as depicted schematically in Fig. 1(b).

Since the biphenyl end group with its extended $\pi$-electron system appeared to be most promising and because of its good insulating properties with respect to the silicon substrate described above, experiments concentrated on that system. Furthermore, the biphenyl SAM covered surface is strongly hydrophobic which limits the possible influence of a water film. The source-drain contact distances were varied between 5 and $10 \mu \mathrm{m}$ and the influence of oxidizing dopants such as $\mathrm{I}_{2}$ was studied.

Lateral conductivity along the end groups could be demonstrated for the first time in the case of $I_{2}$ doping which is shown in Fig. 5 for a 10- and 5- $\mu \mathrm{m}$ source-drain distance. Notice the large difference in conductivity between the undoped and the $I_{2}$-doped biphenyl groups. In addition, the currents increase by nearly two orders of magnitude when the source-drain distance is reduced from 10 to $5 \mu \mathrm{m}$. Since, in both cases, the current values are still very low, the influence of a gate voltage was masked by the existing tunneling currents across the SAM layer. However, it may be argued 

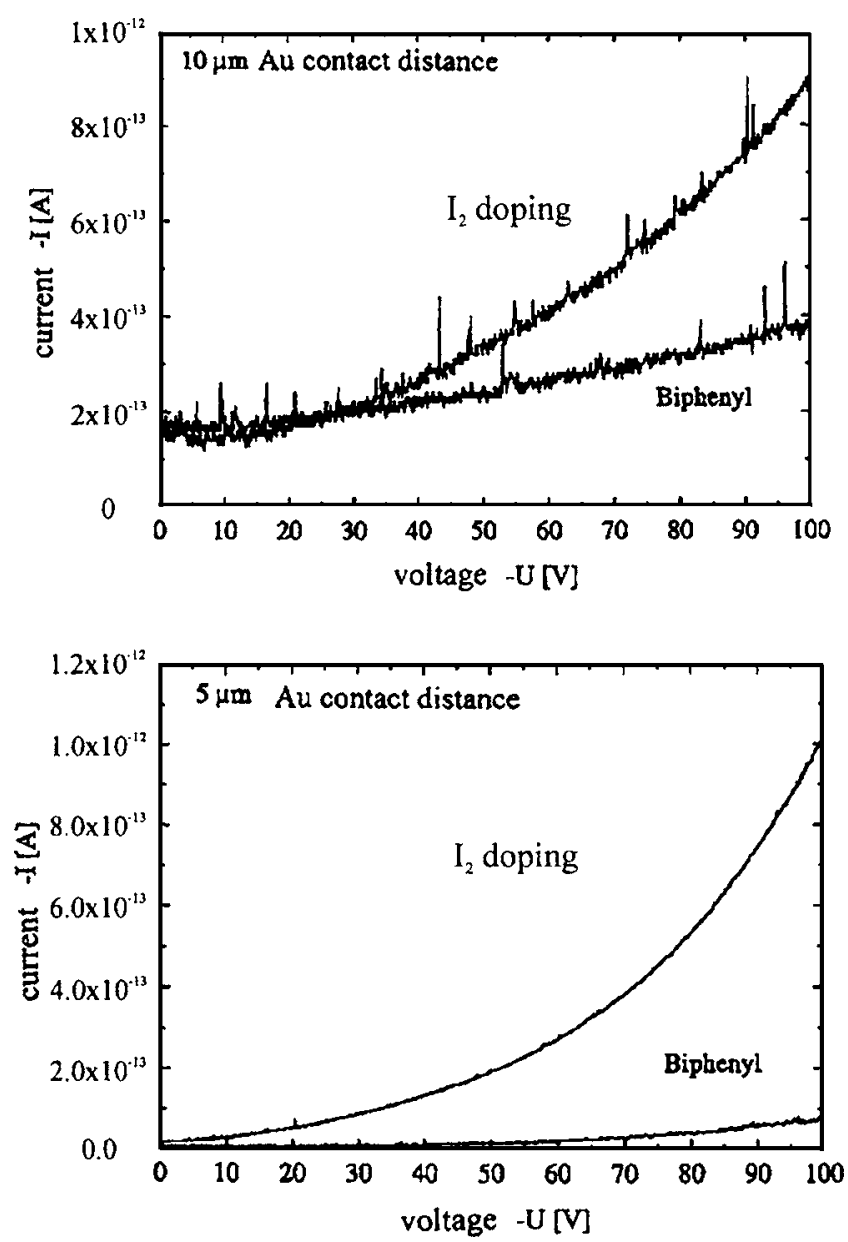

FIG. 5. Current-voltage characteristics demonstrating lateral conduction along the biphenyl end group doped with iodine, for two different sourcedrain contact distances.

that competitive current contributions through a parallel shunt path along the silicon surface including tunneling through the SAM layer would not be influenced by the iodine doping. Therefore, possible parallel shunt currents through the silicon substrate are assumed to be small.

\section{CONCLUSION AND OUTLOOK}

(1) Self-assembling monolayers (SAM) appear to be an interesting approach towards molecular nanostructures. In this paper, SAM layers on silicon surfaces are examined, because of the silicon nanoelectronic potential as a periphery for organic molecular systems.

(2) Through a chemical reaction of a $\mathrm{SiCl}_{3}$ anchor group with a hydroxylated silicon surface, the organic molecules consisting of an alkyl $\left(\mathrm{CH}_{2}\right)_{18}$ chain with different functional end groups were attached to the silicon surface. The surprising result of grazing incidence $\mathrm{x}$-ray reflectivity is that only a two layer model pertaining to two layers with different refractive indices (electron densities) can explain experimental data analysis. One layer of nearly constant thickness is associated with the interface between the hydroxylated silicon surface and the alkyl chain, the other with the actual SAM molecule, i.e., the alkyl chain and the functional end groups.
(3) The electrical properties, exploring the potential for molecular electronic devices, seem to be very promising: SAM layers can serve as excellent gate insulators. ${ }^{16,18}$ Breakdown voltages are very high (typically $16 \mathrm{MV} / \mathrm{cm}$ ), favorably comparing them with the values for $\mathrm{SiO}_{2}$ layers. However, in that case, a conductive active layer such as pentacene ${ }^{27}$ is still needed on top of the SAM.

(4) A lateral in-plane conductivity along the SAM end groups could be demonstrated in the case of an iodinedoped biphenyl end group in an organic field-effect transistor (OFET)-like experimental structure. This opens the possibility to obtain molecular transistors without deposition of an additional conductive active layer. For a still fairly large contact distance of $5 \mu \mathrm{m}$, the in-plane currents are still very small. For a functioning molecular SAM nanotransistor, the source-drain distance has to be reduced considerably, i.e., into the $30-\mathrm{nm}$ range.

\section{ACKNOWLEDGMENTS}

The authors wish to thank F. Baltá Calleja, Madrid, for scientific discussions, and R. García (Madrid, Spain) as well as R. Adhikari (Halle, Germany) for taking AFM images. Financial support of the German Research Association (DFG), Bonn, and the Ministerio de Educación, Cultura y Deporte, Madrid, under Contract No. SAB 2002-2007, is gratefully acknowledged. One of the authors (A.F.) thanks for the financial support of the Dirección General de Investigación (Grant No. BFM 2000-1474), MCYT, Spain. Another author (M.S.M.G.) acknowledges a fellow award by the MCYT (Spain) in the Ramón y Cajal Program.

${ }^{1}$ A. Ulman, An Introduction to Ultrathin Organic Films: From LangmuirBlodgett to Self Assembly (Academic, San Diego, 1991).

${ }^{2}$ F. Schreiber, Prog. Surf. Sci. 65, 151 (2000).

${ }^{3}$ G. E. Poirier and M. J. Tarlov, Langmuir 10, 2853 (1994).

${ }^{4}$ S. Heid, F. Effenberger, K. Bierbaum, and M. Grunze, Langmuir 12, 2118 (1996).

${ }^{5}$ P. Harder, K. Bierbaum, C. Woell, M. Grunze, S. Heid, and F. Effenberger, Langmuir 13, 445 (1997).

${ }^{6}$ K. Bierbaum, M. Kinzler, C. Woell, M. Grunze, G. Haehner, S. Heid, and F. Effenberger, Langmuir 11, 512 (1995).

${ }^{7}$ F. Effenberger, G. Goetz, B. Bidlingmaier, and M. Wezstein, Shore Beach 110, 2651 (1998).

${ }^{8}$ J. S. R. Muenter, thesis, Stuttgart University, 2001.

${ }^{9}$ M. M. Sung, G. J. Kluth, and R. Maboudian, J. Vac. Sci. Technol. A 17, 540 (1999)

${ }^{10}$ S. Seifritz, thesis Stuttgart University, 2001.

${ }^{11}$ K. Bierbaum, M. Grunze, A. A. Baski, L. F. Chi, W. Schrepp, and H. Fuchs, Langmuir 11, 2143 (1995).

${ }^{12}$ R. Moaz, J. Sagiv, D. Degenhardt, H. Moehwald, and P. Quint, Supramol. Sci. 2, 9 (1995)

${ }^{13}$ M. J. Stevens, Langmuir 15, 2773 (1999).

${ }^{14}$ M. Schuetz, thesis, Stuttgart University, 2002.

${ }^{15}$ M. R. Linford and C. E. D. Chidsey, J. Am. Chem. Soc. 115, 12631 (1993).

${ }^{16}$ J. Collet and D. Vuillaume, Appl. Phys. Lett. 73, 2681 (1998).

${ }^{17}$ J. Collet, O. Tharaud, A. Chapoton, and D. Vuillaume, Appl. Phys. Lett. 76, 1941 (2000).

${ }^{18}$ J. Collet, S. Lenfant, D. Vuillaume, O. Bouluossa, F. Rondelez, J. M. Gay, K. Kham, and C. Chevrot, Appl. Phys. Lett. 76, 1339 (2000).

${ }^{19}$ M. M. Heynes et al., Mater. Res. Soc. Symp. Proc. 315, 35 (1993).

${ }^{20}$ N. Tillman, A. Ulman, J. S. Schildkraut, and T. L. Penner, J. Am. Chem. Soc. 110, 6136 (1988) 
${ }^{21}$ B. L. Henke, E. M. Gullikson, and J. C. Davis, At. Data Nucl. Data Tables 54, 181 (1993).

${ }^{22}$ C. Boulas, J. V. Davidovits, F. Rondelez, and D. Vuillaume, Phys. Rev. Lett. 76, 4797 (1996).

${ }^{23}$ D. Vuillaume, C. Boulas, J. Collet, J. V. Davidovitis, and F. Rondelez, Appl. Phys. Lett. 69, 1646 (1996).

${ }^{24} \mathrm{M}$. Rittner (unpublished).
${ }^{25}$ P. Fontaine, D. Goguenheim, D. Deresmes, D. Vuillaume, M. Garet, and F. Rondelez, Appl. Phys. Lett. 62, 2256 (1993).

${ }^{26}$ (unpublished).

${ }^{27}$ M. Halik et al., Nature (London) 431, 963 (2004).

${ }^{28}$ G. Horowitz, Synth. Met. 138, 101 (2003).

${ }^{29}$ K. Sekine, Y. Saito, M. Hirayama, and T. Ohmi, IEEE Trans. Electron Devices 48, 1550 (2000). 